Pacific Journal of Mathematics

CURVATURE FUNCTIONS ON LORENTZ 2-MANIFOLDS 


\title{
CURVATURE FUNCTIONS ON LORENTZ 2-MANIFOLDS
}

\author{
JOHN T. BURNS
}

\begin{abstract}
0. Introduction. This paper deals with the problem of describing those functions which can arise as Gaussian curvatures on 2-dimensional Lorentz manifolds, specifically, the 2-dimensional torus $T^{2}$ and the plane $R^{2}$. It is well known that the only compact connected oriented 2-dimensional manifold which admits a Lorentz metric is the torus $T^{2}$, so restricting attention to $T^{2}$ represents no loss in generality.
\end{abstract}

The technique used is that used by Kazdan and Warner in their study of Gaussian curvatures on Riemannian manifolds. The manifold $M$ is given the standard flat Lorentz metric $g: d s^{2}=d x^{2}-d y^{2}$. Then a given smooth function $K$ will be the Gauss curvature of the Lorentz metric $\bar{g}=e^{2 u} g$ if the function $u$ is a smooth solution of the nonlinear hyperbolic partial differential equation $\square u=u_{x x}-u_{y y}=-K e^{2 u}$. Solving this equation on $T^{2}$ is equivalent to finding a solution $u(x, y)$ in the plane which is periodic in each variable. Although a considerable literature exists on the problem of global periodic solutions of nonlinear hyperbolic partial differential equations, the emphasis is on other types of equations, and our results appear to be new. Our results illustrate a number of significant differences between curvature functions of Riemannian (positive definite) metrics and Lorentz metrics.

Following Kazdan and Warner $[7,8]$, we say that metrics $\bar{g}$ and $g$ on a manifold $M$ are pointwise conformal if $\bar{g}=e^{2 u} g$ for some smooth function $u$ on $M$ and that $\bar{g}$ and $g$ are conformally equivalent if there is a diffeomorphism $\phi$ of $M$ and a smooth function $u$ such that $e^{2 u} g$ is the metric obtained by pulling back $\bar{g}$ under $\phi$, i.e. $\phi^{*}(\bar{g})=e^{2 u} g$. We prescribe a Lorentz metric $g$ on the manifold $M$ and attempt to realize a given function $K$ as the curvature of a Lorentz metric $\bar{g}$ which is pointwise conformal to $g$ or, if that is not possible, which is conformally equivalent to $g$. This approach leads to the problem of solving the nonlinear hyperbolic partial differential equation $\Delta u=-k+K e^{2 u}$, where $k$ and $\Delta$ are the Gaussian curvature and Laplace-Beltrami operator, respectively, in the given metric $g$. (For a derivation of this equation in local coordinates, see Eisenhart [3, p. 90].) The problem of showing that $K$ is the curvature of a metric $\bar{g}$ conformally equivalent to $g$ is precisely that of finding a diffeomorphism $\phi$ of $M$ such that one can solve $\Delta u=-k+(K \circ \phi) e^{2 u}$. For the flat Lorentz metric $g: d s^{2}=$ $d x^{2}-d y^{2}, \Delta u=-\square u=-\left(u_{x x}-u_{y y}\right)$ and $k=0$, so the equation in question is $\square u=u_{x x}-u_{y y}=-K e^{2 u}$. 
In $\S 1$, we study the hyperbolic equation $u_{x x}-u_{y y}=-h e^{u}$, $h \not \equiv 0$. For the corresponding elliptic equation $u_{x x}+u_{y y}=-h e^{u}$, Kazdan and Warner have shown that the conditions (i) $h$ changes sign on $T^{2}$ and (ii) $\int_{T^{2}} h d A<0$, where $d A$ is the area element of the given metric, are necessary and sufficient for the existence of a solution. For the hyperbolic equation, we show that condition (ii) is no longer necessary and that (i) can be replaced by a stronger necessary condition which in turn implies that (i) and (ii) together are no longer sufficient to guarantee a solution.

In $\S 2$, we study the ordinary differential equation $u^{\prime \prime}=-h e^{u}$, where $h(x)$ is a smooth $2 \pi$-periodic function of a single variable. We show that the conditions (i) $h$ changes sign on $[0,2 \pi]$ and (ii) $\int_{0}^{2 \pi} h(x)<0$ are necessary and sufficient for the existence of a $2 \pi$-periodic solution. We use this result to determine which functions of a single variable may be Gaussian curvatures of Lorentz metrics on $T^{2}$. In particular, there exists a Lorentz metric whose curvature is the curvature of the standard torus of revolution imbedded in $R^{3}$ and possessing the induced Riemannian metric.

Finally, in $\S 3$, we study curvatures on $R^{2}$. Our main results are that if either (1) $K$ is a constant function or (2) $K$ is a function with compact support, then $K$ is the curvature of a Lorentz metric $\bar{g}$ pointwise conformal to the flat metric $d x^{2}=d x^{2}-d y^{2}$. We conclude with some further examples which satisfy neither (1) nor (2).

The results of this paper constitute part of the author's Ph.D. dissertation submitted at the University of Minnesota. The author gratefully acknowledges the advice and encouragement of his advisor, Prof. Leon W. Green.

1. Necessary and sufficient conditions. We turn now to the problem of determining those smooth functions on the 2dimensional torus $T^{2}$ which can be the Gaussian curvature of some Lorentz metric. We begin by considering the flat Lorentz metric whose components are $g_{11}=a^{2}, g_{22}=-b^{2}, g_{12}=g_{21}=0$. The area element associated with the metric $g$ will be denoted by $d A_{g}$, and $d A_{g}=$ $|\operatorname{det} g|^{\frac{1}{2}} d x d y=|a b| d x d y$. We will also think of $T^{2}$ as the plane $R^{2}$ with the points which differ by integer multiples of $2 \pi$ identified. A smooth function on $T^{2}$ will then be a function which is smooth on $R^{2}$ and $2 \pi$-periodic in each variable. The equation we wish to study is

$$
\square_{g} u=\left(1 / a^{2}\right) u_{x x}-\left(1 / b^{2}\right) u_{y y}=-h e^{u} .
$$

For equation (1.1), only (i) of the previously mentioned Riemannian necessary conditions remains. Indeed, by integrating both sides of (1.1) 
over $T^{2}$, we obtain the necessary condition that $\int_{T^{2}} h e^{u} d A_{g}=0$. For a smooth function $h$, this implies that $h$ must change sign. To show that condition (ii) is no longer necessary, we first derive an alternate necessary and sufficient condition for (1.1) to have a solution.

Let $C^{\infty}\left(T^{2}\right)$ denote the set of smooth functions on the torus, and let $P$ be the set of smooth functions on $T^{2}$ which never vanish. Define

$$
S=\left\{h \in C^{\infty}\left(T^{2}\right)|\exists f \in P| h=f \square_{g} f-\left(\left(1 / a^{2}\right) f_{x}^{2}-\left(1 / b^{2}\right) f_{y}^{2}\right)\right\} .
$$

The following proposition, whose main usefulness is in the construction of counterexamples to condition (ii), follows from an easy computation using the change of variable $f=e^{-u}$.

Proposition 1.1. The equation $\square_{g} u=-h e^{2 u}$ has a solution $u \in C^{\infty}\left(T^{2}\right)$ if and only if $h \in S$.

We now specialize to the standard flat Lorentz metric $d s^{2}=$ $d x^{2}-d y^{2}$ and use Proposition 1.1 to show that condition (ii) is no longer necessary. Let $v \in C^{\infty}\left(T^{2}\right)$ be such that $\square v=v_{x x}-v_{y y}=0$, and let $h=v_{x}^{2}-v_{y}^{2}$. Then $f(x, y)=\cosh (v(x, y)) \in P \quad$ and $\quad f \square f-\left(f_{x}^{2}-f_{y}^{2}\right)=$ $h$. Hence $h \in S$, and $\square u=-h e^{2 u}$ has a solution $u \in C^{\infty}\left(T^{2}\right)$. In fact, $u(x, y)=\log \operatorname{sech}(v(x, y))$ is a solution. However, from Green's first identity, we have $\int_{T^{2}} h d A=0$.

In the case of a Lorentz metric $d s^{2}=a^{2} d x^{2}-b^{2} d y^{2}$, where $a$ and $b$ are incommensurable, there are no nonzero functions of the form $h=\left(1 / a^{2}\right) v_{x}^{2}-\left(1 / b^{2}\right) v_{y}^{2}$, where $\square_{g} v=0$, because the only solutions of $\square_{g} v=0$ are constants. However, condition (ii) still fails because of

THEOREM 1.2. On the torus $T^{2}$ with flat Lorentz metric $d s^{2}=$ $a^{2} d x^{2}-b^{2} d y^{2}$ and associated area element $d A_{g}$, there exist functions $h \in C^{\infty}\left(T^{2}\right)$ for which $\square_{g} u=-h^{2 u}$ has a solution and either (1) $\int_{T^{2}} h d A_{g}<0$ or $(2) \int_{T^{2}} h d A_{g}>0$.

Proof. (1) Suppose $f \in P, f(x, y)=w(x), w$ not constant. By Proposition 1.1, $h=\left(1 / a^{2}\right)\left(w(x) w^{\prime \prime}(x)-\left(w^{\prime}(x)\right)^{2}\right)$ is a function for which $\square_{g} u=-h e^{2 u}$ has a solution. Integrating by parts, we have

$$
\int_{T^{2}} h d A_{g}=-4 \pi \frac{|a b|^{\frac{1}{2}}}{a^{2}} \int_{0}^{2 \pi}\left(w^{\prime}(x)\right)^{2} d x<0 .
$$

(2) follows by taking $f \in P, f(x, y)=w(y), w$ not constant. 
For the flat Lorentz metric $d s^{2}=d x^{2}-d y^{2}$ with area element $d A$, we show now that the condition that $h$ changes sign should be replaced by a stronger necessary condition which has considerable geometric content. Recall that an isotropic or null geodesic is one whose tangent vector at every point has length zero. For the standard flat metric, the null geodesics are the lines in the rectangle $[0,2 \pi] \times[0,2 \pi]$ with slope +1 or -1 . These geodesics are all closed. Null geodesics are of course a feature peculiar to indefinite metrics, and our new condition states that if $h$ is such that $u_{x x}-u_{y y}=-h e^{u}$ has a solution, then along each null geodesic either $h$ changes sign or $h=0$. To show this, we use the classical change of variable $x=\xi+\eta, y=\xi-\eta$, and let $w(\xi, \eta)=$ $u(x, y)$ and $H(\xi, \eta)=h(x, y)$. Our equation becomes

$$
w_{\xi \eta}=-H e^{w} \text {. }
$$

For convenience, we shall adopt the following slight abuse of notation. If $w(\xi, \eta)$ is $2 \pi$-periodic in $\xi$ and $\eta$, we shall write $w \in C^{\infty}\left(T^{2}\right)$. Then we have the following easy lemma.

LEMMA 1.3. If $H \in C^{\infty}\left(T^{2}\right)$ and $w \in C^{\infty}\left(T^{2}\right)$ is a solution of (1.2), then for each fixed $\xi_{0} \in[0,2 \pi], \int_{0}^{2 \pi} H\left(\xi_{0}, \eta\right) e^{w\left(\xi_{0}, \eta\right)} d \eta=0$, and for each fixed

$$
\eta_{0} \in[0,2 \pi], \int_{0}^{2 \pi} H\left(\xi, \eta_{0}\right) e^{w\left(\xi, \eta_{0}\right)} d \xi=0
$$

Let $\gamma$ be a null geodesic with slope +1 . (The case with slope -1 is similar.) By periodicity, we may think of $\gamma$ as the line segment from $\left(x_{0},-x_{0}\right)$ to $\left(x_{0}+2 \pi,-x_{0}+2 \pi\right)$, where $0 \leq x_{0} \leq 2 \pi$. We consider the line integral $\int_{\gamma} h e^{u}$. Parameterizing this integral with $x$, we obtain

$$
\int_{x_{0}}^{x_{0}+2 \pi} h\left(x, x-2 x_{0}\right) e^{u\left(x, x-2 x_{0}\right)} d x=\int_{0}^{2 \pi} H\left(\xi, \eta_{0}\right) e^{w\left(\xi, \eta_{0}\right)} d \xi=0
$$

by Lemma 1.3. Since $e^{u}>0$, this implies that either $h$ changes sign along $\gamma$ or $h \equiv 0$ along $\gamma$. We state this result formally as

THEOREM 1.4. If $h \in C^{\infty}\left(T^{2}\right)$ and $\square u=-h e^{u}$ has a solution $u \in C^{\infty}\left(T^{2}\right)$, then along each null geodesic either $h$ must change sign or be equal to zero.

An important consequence of Theorem 1.4 is that the Kazdan and Warner conditions (i) and (ii) for the solvability of the elliptic equation 
are not sufficient to guarantee the existence of a solution of the hyperbolic equation. More precisely, we have

THEOREM 1.5. If $h \in C^{\infty}\left(T^{2}\right)$ changes sign on $T^{2}$, then neither (a) $\int_{T^{2}} h d A>0$, nor (b) $\int_{T^{2}} h d A=0$, nor (c) $\int_{T^{2}} h d A<0$ is sufficient to guarantee the existence of a solution $u \in C^{\infty}\left(T^{2}\right)$ of $\square u=-h e^{u}$.

Proof. We prove (b) with (a) and (c) following as immediate consequences. We represent $T^{2}$ as the rectangle $[0,2 \pi] \times[0,2 \pi]$ with opposite sides identified. Let $p_{0}=\left(x_{0}, y_{0}\right)$ denote the point $(\pi / 2,3 \pi / 2)$. Let $D_{1}$ be the open disc centered at $p_{0}$ with radius $r$, where $r<\pi / 4$. Let $D_{2}$ be the open disc centered at $p_{0}$ with radius $r / 2$. For $t \geqq 0$, there is a smooth function $h_{t}$ on $T^{2}$ with the properties

(i) $h_{t}(p)=t$ for $p \in \bar{D}_{2}$

(ii) $-1<h_{t}(p)<t$ for $p \in D_{1}-\bar{D}_{2}$

(iii) $h_{t}(p)=-1$ for $p \in T^{2}-D_{1}$.

Define $f(t)=\int_{T^{2}} h_{t}$. Clearly, $f(0)<0$, and $t_{0}>0$ may be chosen so that $f\left(t_{0}\right)>0$. Hence, there is a $t^{*} \in\left(0, t_{0}\right)$ such that $0=f\left(t^{*}\right)=\int_{T^{2}} h_{t^{*}}$, and, since $t^{*}>0, h_{t^{*}}$ changes sign on $T^{2}$. However, on the null geodesic $y=x, h_{t^{*}}=-1$, and hence $h_{t^{*}}$ does not satisfy the necessary condition of Theorem 1.4. This completes the proof of (b). The function $h_{t}$ provides the proof of (a), and $-h_{t}$ provides the proof for (c).

If $h$ merely changes sign on $T^{2}$, it is possible in the elliptic case to find a diffeomorphism $\Phi$ of $T^{2}$ so that $\int_{T^{2}}(h \circ \Phi) d A<0$, and hence $u_{x x}+u_{y y}=-(h \circ \Phi) e^{u}$ has a solution. This will not be the case with the hyperbolic equation. Thus Theorem 1.5 reveals a fundamental difference between the Riemannian and Lorentzian cases. While it does not rule out the possibility that any $K \in C^{\infty}\left(T^{2}\right)$ which changes sign may be the curvature of some Lorentz metric, it does have the following important consequences which we state as

COROllary 1.6. For an arbitrary $K \in C^{\infty}\left(T^{2}\right)$, the conditions

(i) $K$ changes sign

(ii) $\int_{T^{2}} K d A<0 \quad\left(\right.$ or $\int_{T^{2}} K d A=0$ or $\left.\int_{T^{2}} K d A>0\right)$ are not sufficient to guarantee that $K$ is the curvature of a metric pointwise conformal to the standard flat Lorentz metric.

Corollary 1.7. For $K \in C^{\infty}\left(T^{2}\right)$, the condition that $K$ changes sign 
is not sufficient to guarantee that $K$ is the curvature of a metric conformally equivalent to the standard flat Lorentz metric.

2. Functions of a single variable. In view of the negative results just presented, the determination of any class of functions satisfying easily verifiable conditions sufficient for $\square u=-h e^{u}$ to have a solution on $T^{2}$ is of considerable interest. One such class consists of those functions $h$ of the form $h(x, y)=f(x)$ or $h(x, y)=g(y)$, where $f$ and $g$ are $2 \pi$-periodic functions of a single variable. For convenience we write $h(x, y)=h(x)$ in order to retain our previous notation. If we seek a solution of the form $u=u(x)$, where $u$ is $2 \pi$-periodic in $x$, we arrive at the ordinary differential equation

$$
u^{\prime \prime}=-h e^{u} \text {. }
$$

Integrating both sides of (2.1), we find that $h$ must change sign on the interval $[0,2 \pi]$. Multiplying (2.1) by $e^{-u}$ and integrating by parts gives $\int_{0}^{2 \pi} h d x=-\int_{0}^{2 \pi} e^{-u}\left(u^{\prime}\right)^{2} d x<0$. Let $C^{\infty}\left(S^{1}\right)$ denote the set of all smooth functions defined on $R^{1}$ which are $2 \pi$-periodic, and let $A\left(S^{1}\right)$ be the set of absolutely continuous functions defined on $R^{1}$ which are $2 \pi$-periodic. Using a one dimensional version of the argument given by Kazdan and Warner [7, Theorem 5.3] for the elliptic partial differential equation which arises in the Riemannian case (see also [2]), we may apply standard arguments of the calculus of variations [1, p. 131] to minimize the functional $J(v)=\int_{0}^{2 \pi}\left(v^{\prime}\right)^{2} d x$ over the set of functions

$$
\boldsymbol{B}=\left\{v \in A\left(S^{1}\right) \mid \int_{0}^{2 \pi} h e^{v} d x=0 \text { and } \int_{0}^{2 \pi} v d x=0\right\}
$$

to conclude that the above necessary conditions are also sufficient to guarantee the existence of a solution. Hence we have

THEOREM 2.1 Let $h \in C^{\infty}\left(S^{1}\right)$. Then the conditions (i) $h$ changes sign and (ii) $\int_{0}^{2 \pi} h d x<0$ are necessary and sufficient for the existence of a solution $u \in C^{\infty}\left(S^{1}\right)$ of equation (2.1).

REMARK. Clearly, the equation $u^{\prime \prime}=h e^{u}$ has a $2 \pi$-periodic solution iff only if $h$ changes sign and $\int_{0}^{2 \pi} h>0$.

We now investigate the geometrical consequences of Theorem 2.1. Let $K_{1}$ be any smooth $2 \pi$-periodic function which changes sign on 
$[0,2 \pi]$ and such that $\int_{0}^{2 \pi} K_{1}(t) d t \neq 0$. If $\int_{0}^{2 \pi} K_{1}<0$, let $K(x, y)=$ $K_{1}(x)$. By Theorem 2.1 there is a smooth function $u(x, y)=u(x)$ such that $\square u=-K e^{u}$. If $\int_{0}^{2 \pi} K_{1}>0$, let $K(x, y)=K_{1}(y)$. By the remark after Theorem 2.1, there is a function $u(x, y)=u(y)$ which solves $\square u=-K e^{u}$. Therefore, the indefiniteness of the Lorentz metric $d s^{2}=$ $d x^{2}-d y^{2}$ allows greater freedom in achieving pointwise conformal metrics with prescribed curvature, at least in the case where the curvature $K$ is a function of one variable.

One particularly interesting application of Theorem 2.1 shows that there exists a Lorentz metric $\bar{g}$ on $T^{2}$, pointwise conformal to $d s^{2}=$ $d x^{2}-d y^{2}$, whose curvature $K$ is equal to the curvature of the standard torus of revolution imbedded in $R^{3}$ and possessing the induced Riemannian metric. The Gauss curvature is $K_{1}(t)=(\cos t) / r(R+\mathrm{r} \cos t)$ with $R>r>0\left[6\right.$, p. 109]. $K_{1}$ changes sign and $\int_{0}^{2 \pi} K_{1}(t) d t<0$, so $K(x, y)=$ $K_{1}(x)$ is the curvature of some $\bar{g}$.

To conclude our geometric observations, we analyze the case of a smooth $2 \pi$-periodic function $K_{1}$ which changes sign on $[0,2 \pi]$ and has $\int_{0}^{2 \pi} K_{1}(t) d t=0$. Since $K_{1}$ changes sign, it is negative on some open set, and hence it is possible to find a diffeomorphism $\psi$ of $S^{1}$ such that $\int_{0}^{2 \pi}\left(K_{1} \circ \psi\right) d t<0$. If Id denotes the identity diffeomorphism of $S^{1}$, then $\Phi=\psi \times I d$ defined by $(\psi \times I d)(x, y)=(\psi(x), y)$ is a diffeomorphism of $T^{2}$, and, if we let $K(x, y)=K_{1}(x)$, then by the argument above we can solve $\square u=-(K \circ \Phi) e^{2 u}$. Hence $K$ is the curvature of the metric $\bar{g}=\left(\Phi^{-1}\right)^{*}\left(e^{2 u} g\right)$, where $g$ is the flat Lorentz metric $d s^{2}=d x^{2}-d y^{2}$. We summarize these results in

THEOREM 2.2. Let $K_{1}$ be a smooth $2 \pi$-periodic function of one variable which changes sign on $[0,2 \pi]$. If $\int_{0}^{2 \pi} K_{1}(t) d t<0$, define $K \in C^{\infty}\left(T^{2}\right)$ by $K(x, y)=K_{1}(x)$. If $\int_{0}^{2 \pi} K_{1}(t) d t>0$, define $K \in C^{\infty}\left(T^{2}\right)$ by $K(x, y)=K_{1}(y)$. Then $K$ is the Gaussian curvature of a Lorentz metric $\bar{g}$ pointwise conformal to the flat metric $d s^{2}=d x^{2}-d y^{2}$.

THEOREM 2.3. Let $K_{1}$ be a smooth $2 \pi$-periodic function of one variable which changes sign on $[0,2 \pi]$. Define $K \in C^{\infty}\left(T^{2}\right)$ by $K(x, y)=$ $K_{1}(x)$. Then $K$ is the curvature of some Lorentz metric $\bar{g}$ (conformally equivalent to $\left.d s^{2}=d x^{2}-d y^{2}\right)$.

3. Curvature functions on $\boldsymbol{R}^{2}$. We now turn our attention to the problem of curvature functions on the plane $R^{2}$. If a smooth 
function $K$ on $R^{2}$ is to be the curvature of a Lorentz metric $\bar{g}$ pointwise conformal to the flat metric $d s^{2}=d x^{2}-d y^{2}$, we must find a smooth function $u$ on $R^{2}$ which is a solution of $\square u=-K e^{2 u}$. Our first main result is that if $K$ is any smooth function with compact support, then $K$ is the curvature of such a metric.

To achieve the analysis necessary to obtain the geometric result, we find it convenient to consider the modified equation

$$
w_{\xi \eta}=\epsilon h(\xi, \eta) e^{w}
$$

where $\epsilon$ is a small real number. Let $C^{\infty}\left(R^{2}\right)$ denote the set of smooth functions on $R^{2}$. We shall prove

THEOREM 3.1. If $h \in C^{\infty}\left(R^{2}\right)$ has compact support, then there is an $\epsilon>0$ such that equation (3.1) has a solution $u \in C^{\infty}\left(R^{2}\right)$.

Proof. Since $h$ has compact support, we may assume that Supp $h$ is contained in the open interior of a triangular region $T$ in the $\xi-\eta$ plane with vertices at $(0,0),(L, 0)$, and $(0, L)$. Furthermore, we may assume that the distance (in the ordinary Euclidean metric on $R^{2}$ ) from Supp $h$ to the boundary of $T$ is $2 \delta>0$. For future use, we note in particular that each side of the triangle is the centerline of an open strip of width $2 \delta$, inside of which $h \equiv 0$. We now show that there is an $\epsilon>0$ for which equation (3.1) has a solution $u$ defined on the rectangle $R=\{(\xi, \eta) \mid 0 \leqq$ $\xi \leqq L, 0 \leqq \eta \leqq L\}$ and which is smooth on the interior of $R$ and satisfies the boundary conditions $u(\xi, 0)=0$ for $0 \leqq \xi \leqq L$ and $u(0, \eta)=0$ for $0 \leqq \eta \leqq L$.

Since Supp $h$ is compact and contained in $R$, we may let $M=$ $\max _{(\xi, \eta) \in R}|h(\xi, \eta)| . \quad$ Set $\epsilon=\epsilon_{0} / M L^{2} e$, where $0<\epsilon_{0}<1$. With $u_{0}(\xi, \eta) \equiv$ 0 , define

$$
u_{n+1}(\xi, \eta)=\epsilon \int_{0}^{\eta} \int_{0}^{\xi} h(s, t) \exp \left(u_{n}(s, t)\right) d s d t, \text { for } n \geqq 0 .
$$

By the standard successive approximations argument, the sequence $\left\{u_{n}\right\}$ converges uniformly to a continuous function $u$ which satisfies $u_{\xi \eta}=$ $\epsilon h(\xi, \eta) e^{u}$. Since $h$ is smooth in the interior of the rectangle $R$, so is $u$. Furthermore, $u(\xi, 0)=0$ for $0 \leqq \xi \leqq L$, and $u(0, \eta)=0$ for $0 \leqq \eta \leqq$ $L$. To extend this local solution to the entire plane, observe that along that part of the noncharacteristic curve $C: \xi+\eta=L$ which lies in the interior of $R$, the functions $u$ and $\partial u / \partial n$, the normal derivative of $u$, are smooth. Furthermore, by the construction of $R$, the support of each of these functions along $C$ is contained in the open segment between $(\delta, L-\delta)$ and $(L-\delta, \delta)$. Hence both $u$ and $\partial u / \partial n$ may be extended 
smoothly along the entire curve $C$ by defining them to be identically zero along those parts of $C$ which lie outside $R$. We can then take this data as the Cauchy data for the equation $w_{\xi \eta}=0$. From [4, p. 110-17], we find that the problem is well-posed and hence has a unique solution $v$ which is in $C^{\infty}\left(R^{2}\right)$ since the Cauchy data are smooth. However, if $(\xi, \eta)$ is a point in the strip of width $2 \delta$ along $C$ and within the interior of $R$, then $h(\xi, \eta)=0$, and our local solution $u$ of (3.1) is actually a solution of $w_{\xi \eta}=0$ with the same Cauchy data as $v$. Hence, in this strip $u \equiv$ $v$. Therefore, we may extend our local solution $u$ into the half-plane $\{(\xi, \eta) \mid \eta>L-\xi\}$ by taking $u(\xi, \eta)=v(\xi, \eta)$. For the extension to the remainder of $R^{2}$, we note that in the open strip $(0, \delta) \times(0, L)$ along the $\eta$-axis and in the open strip $(0, L) \times(0, \delta)$ along the $\xi$-axis, our local solution of (3.1) is identically zero. Moreover, the Cauchy data along the curve $C$ are identically equal to zero outside the segment between $(\delta, L-\delta)$ and $(L-\delta, \delta)$. Hence, to extend our local solution $u$ smoothly to the remaining regions of the plane, we may take $u \equiv 0$ in these regions.

Therefore, for some $\epsilon>0$, we have obtained a solution $u \in C^{\infty}\left(R^{2}\right)$ of equation (3.1).

COROLlARY 3.2. If there is an $\epsilon>0$ for which (3.1) has a solution $u \in C^{\infty}\left(R^{2}\right)$, then $w_{\xi \eta}=h(\xi, \eta) e^{w}$ has a solution $w \in C^{\infty}\left(R^{2}\right)$.

Proof. Let $w=u-\log (1 / \epsilon)$.

Corollary 3.3. Let $K \in C^{\infty}\left(R^{2}\right)$ have compact support. Then $\square u=-K e^{2 u}$ has a solution $u \in C^{\infty}\left(R^{2}\right)$.

Proof. Let $h(\xi, \eta)=-2 K(\xi+\eta, \xi-\eta)$ in Corollary 3.2. Then $u(x, y)=\frac{1}{2} w((x+y) / 2,(x-y) / 2)$ is the desired solution.

Corollary 3.4. Any function $K \in C^{\infty}\left(R^{2}\right)$ with compact support is the Gaussian curvature of some Lorentz metric $\bar{g}$ which is pointwise conformal to the standard flat Lorentz metric $d s^{2}=d x^{2}-d y^{2}$.

To establish our second main result, we let $C^{\infty}\left(R^{1}\right)$ denote the set of smooth functions defined on all of $R^{1}$. Then we have

THEOREM 3.5. Let $h(\xi, \eta)=f(\xi) g(\eta)$, where $f, g \in C^{\infty}\left(R^{1}\right)$, and let $\Phi(\xi)$ and $\psi(\eta)$ be any functions in $C^{\infty}\left(R^{1}\right)$ such that $\Phi(\xi) \psi(\eta)>0$ for all $(\xi, \eta) \in R^{2}$. Let $F(\xi)$ and $G(\eta)$ be such that $F^{\prime}(\xi)=f(\xi) \Phi(\xi)$ and $G^{\prime}(\eta)=g(\eta) \psi(\eta)$. Then

$$
w(\xi, \eta)=\log \left(2 \Phi(\xi) \psi(\eta) / \cosh ^{2}(F(\xi)+G(\eta))\right)
$$


is a solution of $w_{\xi \eta}=-h(\xi, \eta) e^{w}=-f(\xi) g(\eta) e^{w}$ defined and smooth on all of $R^{2}$.

Proof. Compute.

COROllaRy 3.6. If $K$ is any constant, then $w_{\xi \eta}=-K e^{w}$ has a smooth solution.

Corollary 3.7. Let $K$ be any constant. Then $K$ is the Gauss curvature of a Lorentz metric $\bar{g}$ pointwise conformal to the flat metric $d s^{2}=d x^{2}-d y^{2}$ on $R^{2}$.

REMARK. This corollary indicates that the situation for Lorentz metrics on $R^{2}$ with prescribed Gauss curvature differs considerably from that for Riemannian metrics. Wittich [9] has shown the nonexistence of a global smooth solution of the elliptic equation $u_{x x}+u_{y y}=e^{u}$. As a consequence, it is impossible to find a Riemannian metric on the entire plane $R^{2}$ which is of constant negative curvature and pointwise conformal to the standard flat Riemannian metric $d s^{2}=d x^{2}+d y^{2}$. By the corollary, this is not the case with Lorentz metrics.

REMARK. The equation $w_{\xi \eta}=e^{w}$ is classical, having apparently been first treated by Liouville [5, p. 97], who gave the solution $e^{w}=$ $2 f^{\prime}(\xi) g^{\prime}(\eta) /\left((f(\xi)+g(\eta))^{2}\right)$ where $f$ and $g$ are arbitrary twice differentiable functions for which the expression is meaningful.

REMARK. By making specific choices of $f$ and $g$ in Theorem 3.5, we may exhibit some interesting curvatures. $K(x, y)=\exp \left(x^{2}+y^{2}\right)$ is a curvature which is everywhere $\geqq 1 . \quad-K$ is a curvature everywhere $\leqq$ -1 . $K(x, y)=x^{2}-y^{2}$ is a curvature which changes sign.

\section{REFERENCES}

1. N. I. Ahiezer, The Calculus of Variations, Translated from the Russian by Aline H. Frink, Blaisdell Publishing Co., New York-London, 1962.

2. Melvyn S. Berger, On Riemannian structures of prescribed Gauss curvature for compact two-dimensional manifolds, J. Differential Geometry, 5 (1971), 325-332.

3. L. P. Eisenhart, Riemannian Geometry, Princeton University Press, Princeton, New Jersey, 1949.

4. P. Garabedian, Partial Differential Equations, John Wiley \& Sons, Inc., New York-London-Sydney, 1964.

5. E. Goursat, Lecons sur l' integration des equations aux derivees partielles du second ordre, Tome I, Hermann, Paris, 1896.

6. D. Gromoll, W. Klingenberg, and W. Meyer, Riemannsche Geometrie im Grossen, Lecture Notes in Mathematics No. 55, Springer-Verlag, Berlin-New York, 1968. 
7. J. Kazdan, and F. Warner, Curvature functions for compact 2-manifolds, Ann. of Math., 99 (1974), 14-47.

8. - Curvature functions for open 2-manifolds, Ann. of Math., 99 (1974), 203-219.

9. H. Wittich, Ganze Lösungen der Differentialgleichung $\Delta u=e^{u}$, Math. Z., 49 (1944), 579-582.

Received December 8, 1976.

UNIVERSITY OF COLORADO

COLORADO SPRINGS, CO 80907 



\section{PACIFIC JOURNAL OF MATHEMATICS \\ EDITORS}

RICHARD ARENS (Managing Editor)

University of California

Los Angeles, CA 90024

\section{R. A. Beaumont \\ University of Washington \\ Seattle, WA 98105}

C. C. MOORE

University of California

Berkeley, CA 94720

\section{J. DUGUNDJI}

Department of Mathematics

University of Southern California

Los Angeles, CA 90007

R. FINN AND J. MILGRAM

Stanford University

Stanford, CA 94305

\section{ASSOCIATE EDITORS}

\section{E. F. BECKENBACH}

B. H. NeUmanN

F. WOLF

K. YoSHIDA

\section{SUPPORTING INSTITUTIONS}

\author{
UNIVERSITY OF BRITISH COLUMBIA \\ CALIFORNIA INSTITUTE OF TECHNOLOGY \\ UNIVERSITY OF CALIFORNIA \\ MONTANA STATE UNIVERSITY \\ UNIVERSITY OF NEVADA \\ NEW MEXICO STATE UNIVERSITY \\ OREGON STATE UNIVERSITY \\ UNIVERSITY OF OREGON \\ OSAKA UNIVERSITY
}

\author{
UNIVERSITY OF SOUTHERN CALIFORNIA \\ STANFORD UNIVERSITY \\ UNIVERSITY OF HAWAII \\ UNIVERSITY OF TOKYO \\ UNIVERSITY OF UTAH \\ WASHINGTON STATE UNIVERSITY \\ UNIVERSITY OF WASHINGTON \\ AMERICAN MATHEMATICAL SOCIETY
}

The Supporting Institutions listed above contribute to the cost of publication of this Journal, but they are not owners or publishers and have no responsibility for its contents or policies.

Mathematical papers intended for publication in the Pacific Journal of Mathematics should be in typed form or offset-reproduced (not dittoed), double spaced with large margins. Underline Greek letters in red, German in green, and script in blue. The first paragraph or two must be capable of being used separately as a synopsis of the entire paper. Items of the bibliography should not be cited there unless absolutely necessary, in which case they must be identified by author and Journal, rather than by item number. Manuscripts, in duplicate, may be sent to any one of the four editors. Please classify according to the scheme of Math. Reviews, Index to Vol. 39. All other communications should be addressed to the managing editor, or Elaine Barth, University of California, Los Angeles, California, 90024.

100 reprints are provided free for each article, only if page charges have been substantially paid. Additional copies may be obtained at cost in multiples of 50 .

The Pacific Journal of Mathematics is issued monthly as of January 1966. Regular subscription rate: $\$ 72.00$ a year (6 Vols., 12 issues). Special rate: $\$ 36.00$ a year to individual members of supporting institutions.

Subscriptions, orders for back numbers, and changes of address should be sent to Pacific Journal of Mathematics, 103 Highland Boulevard, Berkeley, California, 94708.

PUBLISHED BY PACIFIC JOURNAL OF MATHEMATICS, A NON-PROFIT CORPORATION

Printed at Jerusalem Academic Press, POB 2390, Jerusalem, Israel.

Copyright (C) 1977 Pacific Journal of Mathematics All Rights Reserved 


\section{Pacific Journal of Mathematics \\ Vol. 70, No. $2 \quad$ October, 1977}

B. Arazi, A generalization of the Chinese remainder theorem ........... 289

Thomas E. Armstrong, Polyhedrality of infinite dimensional cubes .... . . . 297

Yoav Benyamini, Mary Ellen Rudin and Michael L. Wage, Continuous

images of weakly compact subsets of Banach spaces ............ 309

John Thomas Burns, Curvature functions on Lorentz 2-manifolds ......... 325

Dennis F. De Riggi and Nelson Groh Markley, Shear distality and equicontinuity .................................. 337

Claes Fernström, Rational approximation and the growth of analytic

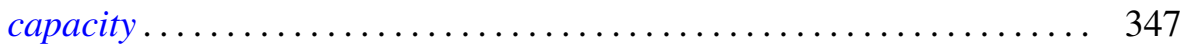

Pál Fischer, On some new generalizations of Shannon's inequality....... 351

Che-Kao Fong, Quasi-affine transforms of subnormal operators ......... 361

Stanley P. Gudder and W. Scruggs, Unbounded representations of

*-algebras........................................ 369

Chen F. King, A note on Drazin inverses .................... 383

Ronald Fred Levy, Countable spaces without points of first countability . . . 391

Eva Lowen-Colebunders, Completeness properties for convergence

spaces ......................................... 401

Calvin Cooper Moore, Square integrable primary representations ....... 413

Stanisław G. Mrówka and Jung-Hsien Tsai, On preservation of

E-compactness ................................ 429

Yoshiomi Nakagami, Essential spectrum $\Gamma(\beta)$ of a dual action on a von

Neumann algebra ................................ 437

L. Alayne Parson, Normal congruence subgroups of the Hecke groups

$G\left(2^{(1 / 2)}\right)$ and $G\left(3^{(1 / 2)}\right)$...

Louis Jackson Ratliff, Jr., On the prime divisors of zero in form rings . . . . 489

Caroline Series, Ergodic actions of product groups .................. 519

Robert O. Stanton, Infinite decomposition bases..................... 549

David A. Stegenga, Sums of invariant subspaces .................. 567 